Скопје, Македонија

\title{
COMMON FIXED POINTS IN b-DISLOCATED METRIC SPACES USING (E.A) PROPERTY
}

\author{
Kastriot Zoto ${ }^{1}$, Ilir Vardhami ${ }^{2}$, Jani Dine ${ }^{3}$ and Arben Isufati ${ }^{4}$
}

\begin{abstract}
In this paper, we prove coincidence and common fixed point results for one a pair of mappings that satisfy the (E.A) property and its generalized variants in the setup of b-dislocated metric spaces. Our results generalize and extend some existing results in the literature.
\end{abstract}

\section{INTRODUCTION}

The study of metric fixed point theory in b-metric space was introduced and studied by Bakhtin [4] and Czerwik [10]. After that a series of papers have been published with interesting results about fixed point and common fixed points for different classes of mappings such as single value and multi valued, involving a single map, two mappings, compatible and weakly compatible mappings in the framework of B-metric spaces. One another generalization is dislocated metric spaces considered by P. Hitzler and A. K. Seda in [5] who introduced this metric as a generalization of usual metric, and generalized the Banach contraction principle on this space. Further many papers has been given as in references $[2,6,7,11,13,14,15]$.

Recently a generalization of b-metric space and dislocated metric space such as $b$-dislocated metric spaces was introduced and studied by N. Hussain et.al [7]. Also in [7] are presented some topological aspects and properties of $b$ dislocated metrics. Subsequently, some fixed point and common fixed point results have been investigated for different types of contractions in these spaces.

On the other hand, (E:A)property was introduced in 2002 by Aamri and Moutaawakil in [18]. Later, some authors employed this concept to obtain some new fixed point results, can see $([19,20,21,22])$.

2010 Mathematics Subject Classification. Primary: 47H10 Secondary: 55M20

Key words and phrases. (E.A) property; (E.A) Like property; b-dislocated metric space; weakly compatible maps; common fixed point. 
In this paper, we prove results for a pair of mappings which satisfy the (E.A) and (E.A) Like property in b-dislocated metric spaces. We generalize some coincidence and fixed point theorems for mappings using the concepts of weakly compatible pair of mappings, as well as by using $\psi$-contractive conditions and linear type in a class of spaces such as b- dislocated metric spaces.

\section{Preliminaries}

Definition 2.1 [6]. Let $X$ be a nonempty set and a mapping $d_{l}: X \times X \rightarrow[0, \infty)$ is called a dislocated metric (or simply $d_{l}$-metric) if the following conditions hold for any $x, y, z \in X$ :

i. If $d_{l}(x, y)=0$, then $x=y$

ii. $\quad d_{l}(x, y)=d_{l}(y, x)$

iii. $\quad d_{l}(x, y) \leq d_{l}(x, z)+d_{l}(z, y)$

The pair $\left(X, d_{l}\right)$ is called a dislocated metric space (or $d$-metric space for short). Note that when $x=y, d_{l}(x, y)$ may not be 0 .

Definition 2.2[8]. Let $X$ be a nonempty set and a mapping $b_{d}: X \times X \rightarrow[0, \infty)$ is called a $b$-dislocated metric (or simply $b_{d}$-dislocated metric) if the following conditions hold for any $x, y, z \in X$ and $s \geq 1$ :

a. If $b_{d}(x, y)=0$, then $x=y$

b. $b_{d}(x, y)=b_{d}(y, x)$

c. $b_{d}(x, y) \leq s\left[b_{d}(x, z)+b_{d}(z, y)\right]$

The pair $\left(X, b_{d}\right)$ is called a $b$-dislocated metric space. And the class of $b$ dislocated metric space is larger than that of dislocated metric spaces, since a $b$ dislocated metric is a dislocated metric when $s=1$.

Example 2.3. If $X=R$, then $d_{l}(x, y)=|x|+|y|$ defines a dislocated metric on $X$.

Definition 2.4 [7]Let $\left(X, b_{d}\right)$ a $b_{d}$-metric space, and $\left\{x_{n}\right\}$ be a sequence of points in $X$. A point $x \in X$ is said to be the limit of the sequence $\left\{x_{n}\right\}$ if 
$\lim _{n \rightarrow \infty} b_{d}\left(x_{n}, x\right)=0$ and we say that the sequence $\left\{x_{n}\right\}$ is $b_{d}$-convergent to $x$ and denote it by $x_{n} \rightarrow x$ as $n \rightarrow \infty$.

The limit of a $b_{d}$-convergent sequence in a $b_{d}$-metric space is unique [8, Proposition 1.27].

Definition 2.5 [7]. A sequence $\left\{x_{n}\right\}$ in a $b_{d}$-metric space $\left(X, b_{d}\right)$ is called a $b_{d}$-Cauchy sequence iff given $\varepsilon>0$, there exists $n_{0} \in N$ such that for all $n, m>n_{0}$, we have $b_{d}\left(x_{n}, x_{m}\right)<\varepsilon$ or $\lim _{n, m \rightarrow \infty} b_{d}\left(x_{n}, x_{m}\right)=0$.

Every $b_{d}$-convergent sequence in a $b_{d}$-metric space is a $b_{d}$-Cauchy sequence.

Definition 2.6 [7]. A $b_{d}$-metric space $\left(X, b_{d}\right)$ is called complete if every $b_{d}$ Cauchy sequence in $X$ is $b_{d}$-convergent.

Definition 2.7 [20]. Let $f$ and $g$ be two self mappings on a metric space $(X, d)$. The mappings $f$ and $g$ are said to be compatible if

$$
\lim _{n \rightarrow \infty} d\left(f g x_{n}, g f x_{n}\right)=\lim _{n \rightarrow \infty} d\left(f x_{n}, g x_{n}\right)=0
$$

whenever $\left\{x_{n}\right\}$ is a sequence in $X$ such that $\lim _{n \rightarrow \infty} f x_{n}=\lim _{n \rightarrow \infty} g x_{n}=z$, for some $z \in X$

Definition 2.8 [23]. Let $f$ and $g$ be self mappings of a set $X$. Then, $f$ and $g$ are said to be weakly compatible if they commute at their coincidence point; that is $f x=g x$ for some $x \in X$ implies $g f x=f g x$.

Some examples in the literature shows that in general a $b$-dislocated metric is not continuous.

Lemma 2.9 [7]. Let $\left(X, b_{d}\right)$ be a $b$-dislocated metric space with parameter $s \geq 1$. Suppose that $\left\{x_{n}\right\}$ and $\left\{y_{n}\right\}$ are $b_{d}$-convergent to $x, y \in X$, respectively. Then we have

$$
\frac{1}{s^{2}} b_{d}(x, y) \leq \lim _{n \rightarrow \infty} \inf b_{d}\left(x_{n}, y_{n}\right) \leq \lim _{n \rightarrow \infty} \sup b_{d}\left(x_{n}, y_{n}\right) \leq s^{2} b_{d}(x, y)
$$

In particular, if $b_{d}(x, y)=0$, then we have $\lim _{n \rightarrow \infty} b_{d}\left(x_{n}, y_{n}\right)=0=b_{d}(x, y)$. 
Moreover, for each $z \in X$, we have

$$
\frac{1}{s} b_{d}(x, z) \leq \lim _{n \rightarrow \infty} \inf b_{d}\left(x_{n}, z\right) \leq \lim _{n \rightarrow \infty} \sup b_{d}\left(x_{n}, z\right) \leq s b_{d}(x, z)
$$

In particular, if $b_{d}(x, z)=0$, then we have $\lim _{n \rightarrow \infty} b_{d}\left(x_{n}, z\right)=0=b_{d}(x, z)$.

Example 2.10. If $X=\mathbb{R}^{+} \cup\{0\}$, then the function $b_{d}(x, y)=(x+y)^{2}$ defines a $b$-dislocated metric on $X$ with parameter $s=2$.

Consistent with $[18,19]$ are the following definitions in a b-dislocated metric space.

Definition 2.11. Let $X$ be a b-dislocated metric space. Selfmaps $f$ and $g$ on $X$ are said to satisfy the (E.A)-property if there exists a sequence $\left\{x_{n}\right\}$ in $X$ such that $\left\{f x_{n}\right\}$ and $\left\{g x_{n}\right\}$ are $b_{d}$ convergent to some $t \in X$ and $b_{d}(t, t)=0$, (equivalently $\left.\lim _{n \rightarrow \infty} b_{d}\left(f x_{n}, t\right)=\lim _{n \rightarrow \infty} b_{d}\left(g x_{n}, t\right)=b_{d}(t, t)=0\right)$.

Definition 2.12. Let $f$ and $g$ be two self-mappings of a $b$-dislocated metric space $\left(X, b_{d}\right)$. We say that $f$ and $g$ satisfy the (E. A) Like property if there exists a sequence $\left(x_{n}\right)$ such that $\left\{f x_{n}\right\}$ and $\left\{g x_{n}\right\}$ are $b_{d}$ convergent to $t$, for some $t \in f(X)$ or $t \in g(X)$, i.e. $t \in f(X) \cup g(X)$ and $b_{d}(t, t)=0$.

Remark. From the definitions 2.9-2.10, it is evident that a pair $(f, g)$ satisfying the (E.A) like property always enjoys the property (E.A) but the implication is not reversible.

Definition 2.13 [6]. Let $f$ and $g$ be two self-mappings on a non-empty set $X$ then,

(1) Any point $x \in X$ is said to be fixed point of $f$ if $f x=x$.

(2) Any point $x \in X$ is called coincidence point of $f$ and $g$ if $f x=g x$, and we called $u=f x=g x$ is a point of coincidence of $f$ and $g$.

(3) A point $x \in X$ is called common fixed point of $f$ and $g$ if $f x=g x=x$. 


\section{MAin RESUlt}

In this section, some common fixed point results for two mappings satisfying "max" type of contractive conditions and by using altering distance functions $\psi \in \Psi$, in the framework of a b-dislocated metric space, are obtained.

Let $\Psi$ denote the set of all continuous and non decreasing functions $\psi:[0, \infty) \rightarrow[0, \infty)$ such that $\psi(t)=0$ iff $t=0$, and we start with the following theorem.

Theorem 3.1 Let $\left(X, b_{d}\right)$ be a b-dislocated -metric space with parameter $s \geq 1$ and $f, g: X \rightarrow X$ are two self mappings such that for all $x, y \in X$, constant $0 \leq c<1$ and $\psi \in \Psi$,

$$
\begin{gathered}
\psi\left(2 s^{2} b_{d}(f x, f y)\right) \leq c \psi\left(\operatorname { m a x } \left\{b_{d}(g x, g y), b_{d}(f x, g x), b_{d}(f y, g y),\right.\right. \\
\left.\left.\frac{b_{d}(f x, g y)+b_{d}(g x, f y)}{2 s}\right\}\right)
\end{gathered}
$$

Suppose that the pair $(f, g)$ satisfies (E.A) Like property in $X$. Then the pair $(f, g)$ has a common point of coincidence in $X$. Moreover if the pair $(f, g)$ is weakly compatible then $f$ and $g$ have a unique common fixed point in $X$.

Proof. Since $f$ and $g$ satisfy the E. A. Like Property therefore exists a sequence $\left\{x_{n}\right\}$ in $X$ such that $\lim _{n \rightarrow \infty} f x_{n}=\lim _{n \rightarrow \infty} g x_{n}=t$ for some $t \in f(X)$ or $g(X)$.

Assume that $\lim _{n \rightarrow \infty} f x_{n}=t \in g(X)$. Therefore, $t=g u$ for some $u \in X$.

From condition (3.1) we have:

$$
\begin{gathered}
\psi\left(2 s^{2} b_{d}\left(f u, f x_{n}\right)\right) \leq c \psi\left(\operatorname { m a x } \left\{b_{d}\left(g u, g x_{n}\right), b_{d}(f u, g u), b_{d}\left(f x_{n}, g x_{n}\right),\right.\right. \\
\left.\left.\frac{b_{d}\left(f u, g x_{n}\right)+b_{d}\left(g u, f x_{n}\right)}{2 s}\right\}\right)
\end{gathered}
$$

Taking the upper limit as $n \rightarrow \infty$ using lemma 2.9 and definition 2.11, we get

$$
\begin{aligned}
\psi\left(2 s b_{d}(f u, t)\right) & =\psi\left(2 s^{2} \frac{1}{s} b_{d}(f u, t)\right) \leq \psi\left(2 s^{2} \lim _{n \rightarrow \infty} \sup b_{d}\left(f u, f x_{n}\right)\right) \\
& \leq c \psi\left(\lim _{n \rightarrow \infty} \sup \max \left\{b_{d}(t, t), b_{d}(f u, t), s b_{d}(t, t), \frac{s b_{d}(f u, t)+b_{d}(t, t)}{2 s}\right\}\right) \\
& \leq c \psi\left(\max \left\{0, b_{d}(f u, t), 0, \frac{b_{d}(f u, t)}{2}\right\}\right)
\end{aligned}
$$

As a result we have,

$$
\psi\left(2 s b_{d}(f u, t)\right) \leq c \psi\left(b_{d}(f u, t)\right) .
$$


By property of $\psi$, since $0<c<1$ and $s \geq 1$ the above inequality implies $\psi\left(b_{d}(f u, t)\right)=0$ that is $f u=t$.

Therefore we have that $u$ is a coincidence point of $f$ and $g(f u=g u=t)$.

The weak compatibility of $f$ and $g$ implies that,

$$
f t=f g u=g f u=g t
$$

Let we show that $t$ is a fixed point of $f$. According to the condition 3.1, consider:

$$
\begin{gathered}
\psi\left(2 s^{2} b_{d}\left(f t, f x_{n}\right)\right) \leq c \psi\left(\operatorname { m a x } \left\{b_{d}\left(g t, g x_{n}\right), b_{d}(f t, g t), b_{d}\left(f x_{n}, g x_{n}\right),\right.\right. \\
\left.\left.\frac{b_{d}\left(f t, g x_{n}\right)+b_{d}\left(g t, f x_{n}\right)}{2 s}\right\}\right)
\end{gathered}
$$

Taking the upper limit as $n \rightarrow \infty$ and using lemma 2.9, we get

$$
\begin{aligned}
\psi\left(2 s b_{d}(f t, t)\right) & =\psi\left(2 s^{2} \frac{1}{s} b_{d}(f t, t)\right) \leq \psi\left(2 s^{2} \lim _{n \rightarrow \infty} \sup b_{d}\left(f t, f x_{n}\right)\right) \\
& \leq c \psi\left(\operatorname { l i m } _ { n \rightarrow \infty } \operatorname { s u p } \operatorname { m a x } \left\{b_{d}\left(g t, g x_{n}\right), b_{d}(f t, g t), b_{d}\left(f x_{n}, g x_{n}\right),\right.\right. \\
& \left.\left.\frac{b_{d}\left(f t, g x_{n}\right)+b_{d}\left(g t, f x_{n}\right)}{2 s}\right\}\right) \\
& \leq c \psi\left(\max \left\{s b_{d}(f t, t), b_{d}(f t, f t), 0, \frac{s b_{d}(f t, t)+0}{2 s}\right\}\right) \\
& \leq c \psi\left(2 s b_{d}(f t, t)\right)
\end{aligned}
$$

This inequality implies $\psi\left(2 s b_{d}(f t, t)\right)=0$, and as result $f t=g t=t$. Hence, $t$ is a common fixed point of $f$ and $g$.

Uniqueness. Let $t \neq t_{1}$ be two common fixed points of the mappings $f$ and $g$.

Then from (3.1) we have:

$$
\begin{aligned}
\psi\left(2 s b_{d}\right. & \left.\left(f t, f t_{1}\right)\right) \leq \psi\left(2 s^{2} b_{d}\left(f t, f t_{1}\right)\right) \\
& \leq c \psi\left(\max \left\{b_{d}\left(g t, g t_{1}\right), b_{d}(f t, g t), b_{d}\left(f t_{1}, g t_{1}\right), \frac{b_{d}\left(f t, g t_{1}\right)+b_{d}\left(g t, f t_{1}\right)}{2 s}\right\}\right) \\
& =c \psi\left(\max \left\{b_{d}\left(t, t_{1}\right), b_{d}(t, t) b_{d}\left(t_{1}, t_{1}\right), \frac{b_{d}\left(t, t_{1}\right)+b_{d}\left(t, t_{1}\right)}{2 s}\right\}\right) \\
& \leq c \psi\left(2 s b_{d}\left(t, t_{1}\right)\right)
\end{aligned}
$$

This inequality implies that $\psi\left(2 s b_{d}\left(t, t_{1}\right)\right)=0$, since $0 \leq c<1$. we get, $t=t_{1}$. Hence the proof is complete.

The following example illustrates theorem.

Example 3.2 Let $X=[0,1]$ and $b_{d}(x, y)=(x+y)^{2}$ for all $x, y \in X$ is a bdislocated metric on $X$. Then $\left(X, b_{d}\right)$ be a b-dislocated metric space. We take the function $\psi(t)=t$ and define the mappings 


$$
f x=\left\{\begin{array}{ll}
\frac{1}{10} x, & \text { if } x \in[0,1) \\
\frac{1}{12}, & \text { if } x=1
\end{array} \quad \text { and } g x=\frac{1}{2} x .\right.
$$

If we consider the sequence $\left\{x_{n}\right\}$, where $x_{n}=\frac{1}{n}$ for all $n \in N$ it is clear that $f$, $g$ satisfy (E.A) Like property $\lim _{n \rightarrow \infty} f x_{n}=\lim _{n \rightarrow \infty} g x_{n}=0$ for $0 \in f(X)$ or $g(X)$.

For $x, y \in[0,1)$ we have

$$
\begin{aligned}
2 s^{2} b_{d}(f x, f y) & =8 b_{d}\left(\frac{1}{10} x, \frac{1}{10} y\right)=8\left(\frac{1}{10} x+\frac{1}{10} y\right)^{2} \\
& =\frac{8}{25}\left(\frac{1}{2} x+\frac{1}{2} y\right)^{2} \\
& \leq \alpha b_{d}(g x, g y)
\end{aligned}
$$

For $y<x=1$ we have

$$
\begin{aligned}
2 s^{2} b_{d}(f 1, f y) & =8 b_{d}\left(\frac{1}{12}, \frac{y}{10}\right)=8\left(\frac{1}{12}+\frac{y}{10}\right)^{2} \leq 8\left(\frac{1}{10}+\frac{y}{10}\right)^{2} \leq \frac{8}{25}\left(\frac{1}{2}+\frac{y}{2}\right)^{2} \\
& =\frac{8}{25} b_{d}(g 1, g y) \leq \alpha b_{d}(g 1, g y)=\alpha b_{d}(g x, g y)
\end{aligned}
$$

For $x<y=1$ we have

$$
\begin{aligned}
2 s^{2} b_{d}(f x, f 1) & =8 b_{d}\left(\frac{x}{10}, \frac{1}{12}\right)=8\left(\frac{x}{10}+\frac{1}{12}\right)^{2} \leq \frac{8}{25}\left(\frac{x}{2}+\frac{1}{2}\right)^{2} \\
& =\frac{8}{25} b_{d}(g x, g 1) \leq \alpha b_{d}(g x, g 1)=\alpha b_{d}(g x, g y)
\end{aligned}
$$

For $y=x=1$ we have

$$
\begin{aligned}
2 s^{2} b_{d}(f 1, f 1) & =8 b_{d}\left(\frac{1}{12}, \frac{1}{12}\right)=8\left(\frac{1}{12}+\frac{1}{12}\right)^{2} \leq \frac{8}{25}\left(\frac{1}{2}+\frac{1}{2}\right)^{2} \\
& =\frac{8}{25} b_{d}(g 1, g 1) \leq \alpha b_{d}(g x, g y)
\end{aligned}
$$

As a result we have that,

$$
\begin{aligned}
2 s^{2} b_{d}(f x, f y) & \leq \frac{8}{25} b_{d}(g x, g y) \\
& \leq c \max \left\{b_{d}(g x, g y), b_{d}(g x, f x), b_{d}(g y, f y), \frac{b_{d}(f x, g y)+b_{d}(f y, g x)}{2 s}\right\}
\end{aligned}
$$

holds for all $x, y \in X, 0 \leq c<\frac{1}{2}$ and obviously $x=0$ is the unique common fixed point of $f$ and $g$.

Corollary 3.3. Let $\left(X, b_{d}\right)$ be a b-dislocated -metric space with parameter $s \geq 1$ and $f, g: X \rightarrow X$ are two self mappings such that for all $x, y \in X$, constant $0 \leq c<1$,

$$
2 s^{2} b_{d}(f x, f y) \leq c \max \left\{b_{d}(g x, g y), b_{d}(f x, g x), b_{d}(f y, g y), \frac{b_{d}(f x, g y)+b_{d}(g x, f y)}{2 s}\right\}
$$


Suppose that the pair $(f, g)$ satisfies (E.A) Like property in $X$. Then the pair $(f, g)$ has a common point of coincidence in $X$. Moreover if the pair $(f, g)$ is weakly compatible then $f$ and $g$ have a unique common fixed point in $X$.

Proof. Taking the altering distance function $\psi(t)=t$ (identity function) in theorem 3.1.

Theorem 3.4. Let $\left(X, b_{d}\right)$ be a complete b-dislocated metric space with parameter $s \geq 1$ and $f, g: X \rightarrow X$ are two self mappings with $f(X) \subseteq g(X)$, such that satisfy

$$
\psi\left(s^{2} b_{d}(f x, f y)\right) \leq c \psi\left(\max \left\{b_{d}(g x, g y), b_{d}(f x, g x), b_{d}(f y, g y), \frac{b_{d}(f x, g y)+b_{d}(g x, f y)}{2 s}\right\}\right)
$$

for all $x, y \in X$, where $0 \leq c<1$ and $\psi \in \Psi$. Suppose that the pair $(f, g)$ satisfies (E.A) property and $g(X)$ is $b_{d}$-closed in $X$. Then the pair $(f, g)$ has a common point of coincidence in $X$. Moreover if the pair $(f, g)$ is weakly compatible then $f$ and $g$ have a unique common fixed point in $X$.

Proof. Since $f$ and g satisfy the E.A. property, therefore there exists a sequence $\left\{x_{n}\right\}$ in $X$ such that $\lim _{n \rightarrow \infty} f x_{n}=\lim _{n \rightarrow \infty} g x_{n}=t$ for some $t \in X$. As $g(X)$ is a $b_{d}$ closed subspace of X; therefore, every convergent sequence of points of $g(X)$ has a limit in $g(X)$. Therefore,

$$
t=\lim _{n \rightarrow \infty} f x_{n}=\lim _{n \rightarrow \infty} g x_{n}=g u \text { for some } u \in X
$$

This implies $t=g u \in g(X)$ and in this conditions the pair $(f, g)$ satisfies (E.A) Like property and the proof follows from theorem 3.1.

Theorem 3.5. Let $\left(X, b_{d}\right)$ be a b-dislocated -metric space with parameter $s \geq 1$ and $f, g: X \rightarrow X$ are two self mappings such that,

$$
s^{2} b_{d}(f x, f y) \leq \alpha b_{d}(g x, f y)+\beta b_{d}(g x, g y)+\gamma b_{d}(g y, f y)+\delta b_{d}(g x, f x)(3.7)
$$

for all $x, y \in X$ where the constants $\alpha, \beta, \gamma, \delta$ are non negative and $0 \leq \alpha+\beta+\gamma+\delta<\frac{1}{2}$.

Suppose that the pair $(f, g)$ satisfies (E.A) Like property in $X$. Then the pair $(f, g)$ has a common point of coincidence in $X$. Moreover if the pair $(f, g)$ is weakly compatible then $f$ and $g$ have a unique common fixed point in $X$. 
Proof. Since $f$ and $g$ satisfy the (E. A.) Like Property, therefore exists a sequence $\left\{x_{n}\right\}$ in $X$ such that $\lim _{n \rightarrow \infty} f x_{n}=\lim _{n \rightarrow \infty} g x_{n}=g u$ for some $u \in X$.

Assume that $\lim _{n \rightarrow \infty} f x_{n}=t \in g(X)$. Therefore, $t=g u$ for some $u \in X$.

From condition (3.7) we have:

$$
s^{2} b_{d}\left(f u, f x_{n}\right) \leq \alpha b_{d}\left(g u, g x_{n}\right)+\beta b_{d}(f u, g u)+\gamma b_{d}\left(f x_{n}, g x_{n}\right)+\delta b_{d}\left(f u, g x_{n}\right)
$$

Taking the upper limit as $n \rightarrow \infty$ in (3.8), and using lemma 2.9 we get

$$
\begin{aligned}
s b_{d}(f u, t) & =s^{2} \cdot \frac{1}{s} b_{d}(f u, t) \leq \alpha \cdot 0+\beta s b_{d}(f u, t)+\gamma \cdot 0+\delta s b_{d}(f u, t) \\
& =(\beta+\delta) s b_{d}(f u, t) \\
& \leq(\alpha+\beta+\gamma+\delta) s b_{d}(f u, t)
\end{aligned}
$$

From this inequality since $0 \leq c<\frac{1}{2}$ and $s \geq 1$ have $b_{d}(t, f u)=0$ implies $f u=t$.

Therefore we have that $u$ is a coincidence point of $f$ and $g(f u=g u=t)$.

The weak compatibility of $f$ and $g$ implies that,

$$
f t=f g u=g f u=g t
$$

Let we show that $t$ is a common fixed point of $f$. According to the condition 3.7, consider:

$$
s^{2} b_{d}\left(f t, f x_{n}\right) \leq \alpha b_{d}\left(g t, g x_{n}\right)+\beta b_{d}(f t, g t)+\gamma b_{d}\left(f x_{n}, g x_{n}\right)+\delta b_{d}\left(f t, g x_{n}\right)
$$

Taking the upper limit as $n \rightarrow \infty$ we get

$$
\begin{aligned}
s b_{d}(f t, t) & =s^{2} \frac{1}{s} b_{d}(f t, t) \leq \alpha s b_{d}(f t, t)+\beta b_{d}(f t, f t)+\gamma \cdot 0+\delta s b_{d}(f t, t) \\
& \leq(\alpha+2 \beta+\gamma+\delta) s b_{d}(f t, t)
\end{aligned}
$$

Since $0 \leq \alpha+\beta+\gamma+\delta<\frac{1}{2}$ and $s \geq 1$ this inequality implies $b_{d}(f t, t)=0$, and as result $f t=g t=t$. Hence, $t$ is a common fixed point of $f$ and $g$.

Corollary 3.6. Let $\left(X, b_{d}\right)$ be a complete b-dislocated metric space with parameter $s \geq 1$ and $f, g: X \rightarrow X$ are two self mappings with $f(X) \subseteq g(X)$, such that satisfy

$$
s^{2} b_{d}(f x, f y) \leq k\left[b_{d}(g x, f y)+b_{d}(g x, g y)+b_{d}(g y, f y)+b_{d}(g x, f x)\right]
$$

for all $x, y \in X$, where the constant $0<k<1$. Suppose that the pair $(f, g)$ satisfies (E.A) property and $g(X)$ is $b_{d}$-closed in $X$. Then the pair $(f, g)$ has a common point of coincidence in $X$. Moreover if the pair $(f, g)$ is weakly compatible then $f$ and $g$ have a unique common fixed point in $X$. 
Theorem 3.7. Let $\left(X, b_{d}\right)$ be a b-dislocated -metric space with parameter $s \geq 1$ and $f, g: X \rightarrow X$ are two self mappings such that,

$$
\begin{aligned}
s^{2} b_{d}(f x, f y) \leq & \alpha\left[b_{d}(f x, g y)+b_{d}(g x, f y)\right]+\beta\left[b_{d}(f x, g y)+b_{d}(g x, g y)\right] \\
& +\gamma\left[b_{d}(g x, f y)+b_{d}(g x, g y)\right]
\end{aligned}
$$

for all $x, y \in X$ where the constants $\alpha, \beta, \gamma, \delta>0$ are non negative and $0 \leq \alpha+\beta+\gamma<\frac{1}{2}$.

Suppose that the pair $(f, g)$ satisfies (E.A) Like property in $X$. Then the pair $(f, g)$ has a common point of coincidence in $X$. Moreover if the pair $(f, g)$ is weakly compatible then $f$ and $g$ have a unique common fixed point in $X$.

Proof. Since $f$ and $g$ satisfy the E. A. Like Property therefore exists a sequence $\left\{x_{n}\right\}$ in $X$ such that $\lim _{n \rightarrow \infty} f x_{n}=\lim _{n \rightarrow \infty} g x_{n}=z \in f(X)$ or $g(X)$.

Assume that $\lim _{n \rightarrow \infty} f x_{n}=z \in g(X)$. Therefore, $z=g u$ for some $u \in X$.

From condition (3.11) we have:

$$
\begin{aligned}
s^{2} d\left(f u, f x_{n}\right) \leq & \alpha\left[d\left(f u, g x_{n}\right)+d\left(g u, f x_{n}\right)\right]+\beta\left[d\left(f u, g x_{n}\right)+d\left(g u, g x_{n}\right)\right] \\
& +\gamma\left[d\left(g u, f x_{n}\right)+d\left(g u, g x_{n}\right)[]\right.
\end{aligned}
$$

Taking limit as $n \rightarrow \infty$, we get

$$
\begin{aligned}
s d(f u, z) & =s^{2} \cdot \frac{1}{s} d(f u, z) \leq \alpha[s d(f u, z)+0]+\beta[s d(f u, z)+0]+\gamma[0+0] \\
& =(\alpha+\beta) d(f u, z) \\
& \leq(2 \alpha+2 \beta+2 \gamma) d(f u, z)
\end{aligned}
$$

From this inequality have

$$
d(f u, z) \leq \frac{2 \alpha+2 \beta+2 \gamma}{s} d(f u, z)
$$

By (3.13) we get $d(f u, z)=0$ since $0 \leq \frac{2 \alpha+2 \beta+2 \gamma}{s}<1$.

By property $d_{2}$ have $f u=z$. Hence $f u=g u=z$. Using the weak compatibility we get $f z=g z$.

Let we show that $f z=z$. Again consider:

$$
\begin{aligned}
d\left(f z, f x_{n}\right) \leq & \alpha\left[d\left(f z, g x_{n}\right)+d\left(g z, f x_{n}\right)\right]+\beta\left[d\left(f z, g x_{n}\right)+d\left(g z, g x_{n}\right)\right] \\
& +\gamma\left[d\left(g z, f x_{n}\right)+d\left(g z, g x_{n}\right)\right]
\end{aligned}
$$

Taking the upper limit as $n \rightarrow \infty$, we get 


$$
\begin{aligned}
s d(f z, z) & =s^{2} \cdot \frac{1}{s} d(f z, z) \leq \alpha[s d(f z, z)+s d(g z, z)]+\beta[s d(f z, z)+s d(g z, z)] \\
& +\gamma[d(g z, z)+d(g z, z)] \\
& =\alpha[s d(f z, z)+s d(f z, z)]+\beta[s d(f z, z)+s d(f z, z)] \\
& +\gamma[s d(f z, z)+s d(f z, z)] \\
& \leq(2 \alpha+2 \beta+2 \gamma) s d(f z, z)
\end{aligned}
$$

From this we have $d(f z, z)=0$ since $0 \leq \alpha+\beta+\gamma<\frac{1}{2}$. Therefore $d(f z, z)=0$ $\Rightarrow f z=z$.

So $f z=z=g z$. Hence, $z$ is a common fixed point of $f$ and $g$.

Uniqueness. Clearly, as in theorem 3.1 we can show that fixed point is unique.

Remark 3.8 As a consequence of theorem 3.1 and 3.3 for taking

1) the parameter $s=1$

2) the parameter $s=1$ and the identity mapping $f x=x$

3) the parameter $s=1$ and the function $\psi(t)=t$;

we can establish many other corollaries in the setting of dislocated metric spaces

\section{COMPETING INTERESTS}

Authors have declared that no competing interests exist.

\section{References}

[1] C. T. Aage, J. N. Salunke, The results on fixed points in dislocated and dislocated quasi-metric space, Appl. Math. Sci.,2(59), (2008), 2941-2948.

[2] C. T. Aage, J. N. Salunke, Some results of fixed point theorem in dislocated quasi-metric spaces, Bulletin of Marathwada Mathematical Society, 9(2008), 1-5

[3] A. Beiranvand, S. Moradi, M. Omid, H. Pazandeh, Two fixed point theorems for special mapping, arXiv:0903.1504v1 [math.FA].

[4] I. A. Bakhtin, The contraction mapping principle in quasimetric spaces, Funct. Anal., Unianowsk Gos. Ped. Inst. 30, (1989), 26-37

[5] P. Hitzler, A. K. Seda, Dislocated topologies, J. Electr. Engin, 51(12/S):3:7, 2000. 
[6] R. Shrivastava, Z. K. Ansari and M. Sharma, Some results on Fixed Points in Dislocated and Dislocated Quasi-Metric Spaces, Journal of Advanced Studies in Topology, Vol. 3, No.1, (2012)

[7] N. Hussain, J. R. Roshan, V. Parvaneh and M. Abbas, Common fixed point results for weak contractive mappings in ordered b-dislocated metric spaces with applications, Journal of inequalities and Applications, 1/486, (2013)

[8] M. A. Kutbi, M. Arshad, J. Ahmad, A. Azam, Generalized common fixed point results with applications, Abstract and Applied Analysis, volume 2014, article ID 363925, 7 pages

[9] S. Czerwik, Contraction mappings in b-metric spaces. Acta Math. Inform. Univ. Ostrav. 1, 5-11 (1993)

[10] L. B. Ciric, A generalization of Banach's contraction principle, Prooceedings of the American Mathematical Society, vol. 45, (1974), 267273.

[11] M. Arshad, A. Shoaib and I. Beg, Fixed point of a pair of contractive dominated mappings on a closed ball in an ordered dislocated metric space, Fixed point theory and applications, vol. 2013, article 115, 2013

[12] M. A. Alghmandi, N. Hussain and P. Salimi, Fixed point and coupled fixed point theorems on b-metric-like spaces, Journal of inequalities and applications, vol. 2013, article 402, 2013

[13] M. Arshad, A. Shoaib and P. Vetro; Common fixed points of a pair of Hardy Rogers type mappings on a closed ball in ordered dislocated metric spaces, Journal of function spaces and applications, vol 2013, article id 638181

[14] R. Yijie, L. Junlei and Y. Yanrong, Common fixed point theorems for nonlinear contractive mappings in dislocated metric spaces, Abstract and Applied Analysis vol 2013, article id 483059.

[15] K. Zoto, P. S. Kumari and E. Hoxha, Some Fixed Point Theorems and Cyclic Contractions in Dislocated and Dislocated Quasi-Metric Spaces, American Journal of Numerical Analysis, 2.3 (2014), 79-84.

[16] M. Kir and H. Kiziltunc, On Some Well Known Fixed Point Theorems in b-Metric Spaces, Turkish Journal of Analysis and Number Theory, 1.1 (2013), 13-16.

[17] M. P. Kumar, S. Sachdeva and S. K. Banerjee, Some Fixed Point Theorems in b-metric Space, Turkish Journal of Analysis and Number Theory 2.1 (2014), 19-22. 
[18] M. Aamri and D. El Moutawakil Some new common fixed point theorems under strict contractive conditions, J. Math. Anal. Appl. 270, 181-188, 2002.

[19] K. Wadhwa, H. Dubey and R. Jain, Impact of E. A. Like property on common fixed point theorems in fuzzy metric spaces. J. Adv. Stud. Topology 3 (1) (2012), 52-59.

[20] I. Beg nad M. Abbas, Coincidence and common fixed points of noncompatible maps. J. Appl. Math. Inform. 29(3-4), 743-752 (2011).

[21] Sh. Cho and J. S. Bae, Common fixed point theorems for mappings satisfying property (E.A) on cone metric spaces. Math. Comput. Model. 53, 945-951 (2011)

[22] K. Zoto, A. Isufati and P. S. Kumari, Fixed Point Results and E. A Property in Dislocated and Dislocated Quasi- metric Spaces. Turkish Journal of Analysis and Number Theory 3.1 (2015): 24-29.

[23] G. Jungck, Compatible mappings and common fixed points, Int. J. Math. Math. Sci., ( 1986), 771-779.

${ }^{1,3,4)}$ Faculty of Natural Sciences, University of Gjirokastra, Gjirokastra, Albania E-mail address: zotokastriot@yahoo.com

${ }^{2)}$ Faculty of Natural Sciences, University of Tirana, Tirana, Albania 